Sharif University of Technology
Scientia Iranica
Transactions E: Industrial Engineering
SCIENTIA

\title{
Robust parameter design based on response surface model considering measurement errors
}

\author{
F. Wu, J. Wang, and Y. Ma* \\ School of Economics and Management, Nanjing University of Science and Technology, Nanjing, Jiangsu 210094, China.
}

Received 6 June 2018; received in revised form 11 October 2018; accepted 21 December 2019

\author{
KEYWORDS \\ Robust parameter \\ design; \\ Response surface \\ model; \\ Measurement errors; \\ Unbiased estimator; \\ Quality loss.
}

\begin{abstract}
Response surface is an effective method for robust parameter design. Previous response surface methodologies assume that independent variables are measured without errors. However, this assumption might be violated due to the low capability of the measurement system. The present study employs the response surface method for robust parameter design with measurement errors in variables. In addition, an unbiased estimator is presented in the presence of some measurement errors and an optimal setting which is determined to minimize the expected quadratic loss. An example is then illustrated to verify the effectiveness of the proposed approach. The results showed that the proposed method could achieve better operating conditions, despite measurement errors, than the conventional method.

(C) 2021 Sharif University of Technology. All rights reserved.
\end{abstract}

\section{Introduction}

Robust parameter design (also known as parameter design) is a quality improvement technique proposed by Taguchi $[1,2]$, which is a cost-effective approach to reducing variations of products and processes. As summarized by Nair [3] and Phadke [4], Taguchi classified the inputs to the system into two groups: control variables $\mathbf{x}$ and noise variables $\mathbf{z}$. While the former can be easily controlled and manipulated, the latter are difficult, expensive or impossible to control during the normal design or production process. Here, $y$ denotes the response which is actually a quality characteristic that measures the output performance of the system. There could be many combinations or settings of $\mathbf{x}$ where the system can produce the desired level of $y$ (also called the target) on average. Among these, there are also some settings based on which the system becomes insensitive to the effect of the noise

\footnotetext{
*. Corresponding author. Tel.: +86-02584303268
} E-mail address: yzma-2004@163.com (Y. Ma) variables $\mathbf{z}$. Variations in $\mathbf{z}$ during the manufacturing process contribute to more unwanted variations into the system. The basic idea of parameter design lies in selecting the levels of control variables so as to achieve robustness or insensitivity to the noise variation $\mathbf{z}$. This is done by exploiting interactions between the control and noise variables. A traditional approach to the study of robust parameter design is the signalto-noise ratio approach [5], which combines the mean with variance. Although this approach can mitigate the deleterious effects of noise variables, its performance is still limited because it ignores the interactions among the control variables, leading to invalid results sometimes. Robust design problems can be handled using a response surface approach such as interactions between control variables [6]. Response surface model approach establishes a relationship between process responses and process variables containing control variables, noise variables, and interactions between control variables and noise variables. The control variables are set by minimizing the quality loss function, which contains deviation from the target and variance (see, for example $[7-10])$.

To improve the product quality, the first issue is 
to identify process variables that potentially affect the product quality characteristics. Once the influential variables are identified, the next step is to seek appropriate settings for the control variables to optimize process output and improve product quality. The product quality data are obtained through measurements associated with both the design and production stages. Zhai et al. [11] pointed out that measurement errors would occur often inevitably due to various factors such as human errors and limited precision of the measurement device. Gauge measurement errors have been extensively discussed in the literature (e.g., see $[12,13]$. Loken and Gelman [14] found that measurement error added noise to predictions, increased uncertainty in parameter estimates, and made it much more difficult to discover new phenomena. Feng et al. [15] maintained that all measurement processes were subject to a certain degree of uncertainty, emphasizing that measurement result should be reported with a quantitative statement of its uncertainty. The classical theory of robust design assumes that the independent variables are measured without errors. Giovagnoli and Romano [16] introduced a modification to the dual response surface modeling, which incorporates the option of stochastically simulating some of the noise variables when their probabilistic behavior is known. They applied the method to the design of a highprecision optical profilometer and insisted that their method was suitable for designing complex measurement systems. In practice, however, the measurement system is usually not precise enough. A firm needs to design the experiment, analyze the data in the design stage, and maintain the level of control variables in the production stage. Measurement errors can occur in design or production stages, which may not be recognized by the design or production engineers. The effect of errors at the factor levels on the statistical properties of the parameters obtained from two-level factorial and fractional factorial designs was first studied by Box [17]. Draper and Beggs [18] measured the robustness of experimental designs to errors at the factor levels by the sum of squared differences between the observed and predicted values. However, they also recognized that finding an analytical proof for any optimality conditions in the presence of two or more factors was very difficult and, also, recommended searching numerically for a solution. Steiner and Hamada [19] assumed that during regular production, some measurement errors might occur, but negligible measurement errors would emerge during the design process. Under this assumption, the response model derived from the experimental results is unaffected by measurement errors. Donev [20] focused on the statistical properties of experimental designs in which the factor levels could not be set precisely and then proposed that the criterion of D-optimality should be based on the inverse of the information matrix. They recognized that errors in variables could affect the mean and the variance of responses. In the D-optimality, the effect of noise variables is ignored. Moreover, measurement errors have a significant influence on the variance of responses in the fitted model. Zhong et al. [21] pointed that the errors in measuring noise variables had a significant influence on process responses. Ardakani [22] found that error in noise variables caused poor estimation of the response model, which would consequently affect the optimal setting of the control variables. However, they did not consider measurement errors in the control variables. Over the past years, the problem of estimating unknown parameters with measurement errors has been extensively discussed in the literature (e.g., see [23-26]). Nevertheless, it has not received enough attention. Design engineers usually do not consider measurement errors. Since measurement errors are not ignorable in model fitting and optimization, it is important to investigate the impacts of modeling and measurement errors on the performance of robust parameter design, as well as further optimization of the control variables, to improve the robustness to measurement errors.

The purpose of this paper is first to analyze the influence of the measurement errors on the response surface modeling and optimization and, then, to explore the best setting of control variables in this situation. Specifically, the next section presents the response surface model for robust parameter design. The effect of measurement errors on response surface model is analyzed in Section 3 . Section 4 presents how to optimize the control variables setting under measurement errors. The optimization scheme is illustrated with an example in Section 5. Concluding remarks are given in the final section.

\section{Response surface model to robust parameter design}

Welch et al. [27] proposed a single response surface model that utilizes control variables and noise variables. A general statistical model can be expressed as:

$$
y=f(\mathbf{x}, \mathbf{z})+\varepsilon .
$$

In Eq. (1), $\mathbf{x}$ and $\mathbf{z}$ denote the vectors of control and noise variables; $\varepsilon$ is a term representing other sources of variability not accounted for in $f$.

The regression model in Eq. (1) is often described including linear, quadratic, and interaction terms. A second-order statistical model with the main effects and control-by-noise interactions is used:

$$
y=\beta_{\mathbf{0}}+\beta_{\mathbf{1}}^{\mathbf{T}} \mathbf{x}+\beta_{2}^{\mathbf{T}} \mathbf{z}+\mathbf{x}^{\mathbf{T}} \mathbf{B}_{1} \mathbf{x}+\mathbf{x}^{\mathbf{T}} \mathbf{B}_{2} \mathbf{z}+\varepsilon .
$$


In Eq. (2), $\mathbf{x}=\left(x_{1}, x_{2}, \cdots, x_{p}\right)^{T}, \mathbf{z}=\left(z_{1}, z_{2}, \cdots, z_{q}\right)^{T}$, and other vectors and matrices are of appropriate dimensions, i.e.:

$$
\begin{aligned}
& \boldsymbol{\beta}_{1}=\left(\beta_{11}, \beta_{12}, \cdots, \beta_{1 p}\right)^{T}, \\
& \boldsymbol{\beta}_{2}=\left(\beta_{21}, \beta_{22}, \cdots, \beta_{2 q}\right)^{T}, \\
& \mathbf{B}_{1}=\left(\begin{array}{cccc}
B_{1_{11}} & 1 / 2 B_{1_{12}} & \cdots & 1 / 2 B_{1_{1 p}} \\
1 / 2 B_{1_{12}} & B_{1_{22}} & \cdots & 1 / 2 B_{1_{2 p}} \\
\vdots & \vdots & \ddots & \vdots \\
1 / 2 B_{1_{1 p}} & \cdots & \cdots & B_{1_{p p}}
\end{array}\right) \\
& \mathbf{B}_{2}=\left(\begin{array}{cccc}
B_{2_{11}} & 1 / 2 B_{2_{12}} & \cdots & 1 / 2 B_{2_{q 1}} \\
1 / 2 B_{2_{21}} & B_{2_{22}} & \cdots & 1 / 2 B_{2_{q 2}} \\
\vdots & \vdots & \ddots & \vdots \\
1 / 2 B_{2_{q 1}} & \cdots & \cdots & B_{2_{q p}}
\end{array}\right) .
\end{aligned}
$$

It needs to be noted that the regression model should be built using significant terms. In general, the model in Eq. (2) is fairly practicable in many engineering applications, which assumes the following:

a) The product system performance is time-invariant. That is, the real-life manufacturing process is stable and the model parameters ( $\boldsymbol{\beta}$ 's and B's) do not vary with time.

b) $\mathbf{z}$ and $\varepsilon$ are independent of each other with the mean of $\mathbf{z}$ and $\varepsilon$ being 0 and the variance of $\mathbf{z}$ and $\varepsilon$ being $\sum_{z}$ and $\sigma_{\varepsilon}^{2}$, respectively. The regression errors $(\varepsilon)$ are independently and identically distributed.

The model in Eq. (2) can be rewritten as follows:

$$
y=g(\mathbf{x}, \mathbf{z})^{T} \theta+\varepsilon,
$$

where we let:

$$
\begin{aligned}
g(\mathbf{x}, \mathbf{z})= & \left(1, x_{1}, x_{2}, \cdots, x_{p}, z_{1}, z_{2}, \cdots, z_{q},\right. \\
& x_{1} x_{1}, x_{1} x_{2}, x_{1} x_{3}, \cdots, x_{p}, x_{1} z_{1}, x_{1} z_{2}, \\
& \left.\cdots, x_{p} z_{q}\right)^{T},
\end{aligned}
$$

and:

$$
\begin{aligned}
\boldsymbol{\theta}= & \left(\beta_{0}, \beta_{11}, \beta_{12}, \cdots, \beta_{1 p}, \beta_{21}, \beta_{22}, \cdots, \beta_{2 q},\right. \\
& \left.B_{1_{11}}, B_{1_{12}}, \cdots, B_{1_{p p}}, B_{2_{11}}, B_{2_{12}}, \cdots B_{2_{p q}}\right)^{T} .
\end{aligned}
$$

The linear regression coefficients $\boldsymbol{\theta}$ and the error variance $\sigma_{\varepsilon}^{2}$ can be estimated using the data obtained from the experiment by the least squares estimator:

$$
\hat{\theta}=\left(\mathbf{X}^{T} \mathbf{X}\right)^{-1} \mathbf{X}^{T} \mathbf{y}
$$

and:

$$
\hat{\sigma}_{\varepsilon}^{2}=\frac{S S E}{n-p-q-\frac{p(p+1)}{2}-p q-1},
$$

where $\mathbf{X}=\left(g\left(\mathbf{x}_{1}, \mathbf{z}_{1}\right), g\left(\mathbf{x}_{2}, \mathbf{z}_{2}\right), \cdots, g\left(\mathbf{x}_{n}, \mathbf{z}_{n}\right)\right)^{T}, \mathbf{y}=$ $\left(y_{1}, y_{2}, \cdots, y_{n}\right)$ and SSE denotes the sum of the squared errors, that is, $S S E=(\mathbf{y}-\mathbf{X} \hat{\theta})^{T}(\mathbf{y}-\mathbf{X} \hat{\theta})$.

The model proposed by Welch et al. [27] could be used to formulate dual response surfaces (see, for example [28-30]. The choice of optimum setting $\mathbf{x}^{*}$ could be obtained via the joint exploration of the response surfaces generated by the mean and variance of the response. The model for the location response (the mean response) is found by taking the expectation of $f(\mathbf{x}, \mathbf{z})$ as follows:

$$
\mu=E_{\varepsilon, z}[f(\mathbf{x}, \mathbf{z})]=\beta_{\mathbf{0}}+\boldsymbol{\beta}_{1}^{T} \mathbf{x}+\mathbf{x}^{T} \mathbf{B}_{1} \mathbf{x} .
$$

The dispersion response (variance) of $f(\mathbf{x}, \mathbf{z})$ in Model (2) is given by:

$$
\begin{aligned}
\sigma^{2} & =\operatorname{Var}_{\varepsilon, z}[f(\mathbf{x}, \mathbf{z})] \\
& =\left(\boldsymbol{\beta}_{2}^{T}+\mathbf{x}^{T} \mathbf{B}_{2}\right) \Sigma_{z}\left(\boldsymbol{\beta}_{2}^{T}+\mathbf{x}^{T} \mathbf{B}_{2}\right)^{T}+\sigma_{\varepsilon}^{2} .
\end{aligned}
$$

An objective function must be defined to obtain the optimal setting of the control variables. Ames et al. [31] and Murphy et al. [32] studied different types of loss functions. One of the most commonly used loss functions is the quadratic loss function. There are three types of quadratic loss function. Eq. (8) gives the quadratic loss function for a nominal-the-best quality characteristic $y$ where the deviation on either side of the target value $\tau$ is undesirable:

$$
L=K(y-\tau)^{2}
$$

where $K$ is an economic coefficient. The quadratic loss function considers off-target penalty and variance by measuring the deviation between the response and target values. Eq. (9) is the quadratic loss function for a smaller-the-better quality characteristic, which can be obtained by substituting $\tau=0$ in Eq. (8):

$$
L=K y^{2} \text {. }
$$

For the larger-the-better quality characteristic $y$ which has positive values, making $y$ larger is equivalent to making $y^{\prime}=1 / y$ smaller. Thus, a larger-the-better type quality loss function can be used by replacing $\mathrm{y}$ with its reciprocal value $y^{\prime}=1 / y$ as:

$$
L=K y^{\prime 2} \text {. }
$$

Eqs. (9) and (10) represent well-tested functions to calculate quality loss for smaller-the-better and largerthe-better quality characteristics when the quality characteristic does not take negative and zero values. 
For the larger-the-better quality characteristic, the response model should be built based on the relationship between new quality characteristic and process input variables (control variables $\mathbf{x}$ and noise variables $\mathbf{z}$ ).

Based on the quality loss function, expected quality loss can be computed:

$$
J(\mathbf{x})=E(L)=\left\{\begin{array}{l}
K\left[\left(\mu_{y}-\tau\right)^{2}+\sigma_{y}^{2}\right] \\
K\left[\mu_{y}^{2}+\sigma_{y}^{2}\right] \\
K\left[\mu_{y^{\prime}}^{2}+\sigma_{y^{\prime}}^{2}\right]
\end{array}\right.
$$

The economic coefficient $K$ is assumed to be 1 in finding the optimal setting of control variables 1 without loss of generality. Then, the expected quality loss will be simple as follows:

$$
J(\mathbf{x})=E(L)=\left\{\begin{array}{lc}
\left(\mu_{y}-\tau\right)^{2}+\sigma_{y}^{2} & (\mathrm{NTB}) \\
\mu_{y}^{2}+\sigma_{y}^{2} & (\mathrm{STB}) \\
\mu_{y^{\prime}}^{2}+\sigma_{y^{\prime}}^{2} & (\mathrm{LTB})
\end{array}\right.
$$

The optimal setting of control variables can be derived by minimizing the expected loss in Eq. (12):

$$
\mathbf{x}^{*}=\min _{\mathbf{x} \in \Theta} \mathrm{J}(\mathbf{x})
$$

where $\Theta$ is the region of control variables. The optimization procedures are illustrated with an example in Section 4 .

\section{Response surface model with measurement errors}

Response surface models for robust parameter design are widely implemented by engineers to improve product or process quality. However, if the measurement of data collection is followed by errors, they do not represent the true values of the variables of the product or process being measured. Therefore, it is important to perform a valid measurement analysis in advance to ensure the accuracy and precision of the data collected. Repeatability and Reproducibility (R\&R) are the most basic concepts used for the identification of the variation of the measurement system [6]. Gauge R\&R addresses the magnitude of errors in a measurement system. The variance of the errors in a measurement system can be easily calculated by statistical software.

As mentioned in the first section, there often exist measurement errors in practical situations. If there are some measurement errors occurring in the product or process design stage, the data obtained from experiments will be imprecise. Assume that Eq. (3) is the actual form of the regression model. In an experiment employed to estimate the relationship, suppose that one observes the following:

$$
\left\{\begin{array}{l}
\tilde{y}=y+v \\
(\tilde{\mathbf{x}}, \tilde{\mathbf{z}})=(\mathbf{x}, \mathbf{z})+\left(\mathbf{w}_{\mathbf{x}}, \mathbf{w}_{\mathbf{z}}\right)
\end{array}\right.
$$

In $\mathrm{Eq} .(14), y, \mathbf{x}$, and $\mathbf{z}$ denote error-free response, control, and noise variables, and $v$ and $\mathbf{w}$ denote the measurement errors of the corresponding variables. It is assumed that the measurement errors follow the assumptions:

$$
\left[\begin{array}{l}
v \\
\mathbf{w}
\end{array}\right] \sim \operatorname{MVN}\left\{\mathbf{0},\left[\begin{array}{cc}
\sigma_{v}^{2} & 0 \\
0 & \Sigma_{\mathbf{w}}
\end{array}\right]\right\}
$$

where MVN reads 'multivariate normal' and $\Sigma_{\mathbf{w}}$ is a $(p+q) \times(p+q)$ covariance matrix of measurement errors vector w. Note that $v$ is assumed to be independent of each component of $\mathbf{w}$ and components of $\mathbf{w}$ are independent of each other, i.e., $\boldsymbol{\Sigma}_{\mathbf{w}}=$ $\operatorname{diag}\left(\sigma_{w_{x_{1}}}^{2}, \sigma_{w_{x_{2}}}^{2}, \cdots, \sigma_{w_{x_{p}}}^{2}\right)$. However, one can only observe the experimental variables $\tilde{y}$ and $g(\tilde{\mathbf{x}}, \tilde{\mathbf{z}})$ with the relationship $y=g(\mathbf{x}, \mathbf{z})^{T} \theta+\varepsilon$. The Ordinary Least Squares (OLS) estimator $\boldsymbol{\theta}$ based on the observed variables is:

$$
\hat{\boldsymbol{\theta}}=\left(\tilde{\mathbf{X}}{ }^{T} \tilde{\mathbf{X}}\right)^{-1} \tilde{\mathbf{X}}^{T} \mathbf{X} \theta+\left(\tilde{\mathbf{X}}^{T} \tilde{\mathbf{X}}\right)^{-1} \tilde{\mathbf{X}}^{T} \varepsilon
$$

where $\mathbf{X}=\left(g\left(\mathbf{x}_{1}, \mathbf{z}_{1}\right), g\left(\mathbf{x}_{2}, \mathbf{z}_{2}\right), \cdots, g\left(\mathbf{x}_{n}, \mathbf{z}_{n}\right)\right)^{T}$ and $\tilde{\mathbf{X}}=\left(g\left(\tilde{\mathbf{x}}_{1}, \tilde{\mathbf{z}}_{1}\right), g\left(\tilde{\mathbf{x}}_{2}, \tilde{\mathbf{z}}_{2}\right), \cdots, g\left(\tilde{\mathbf{x}}_{n}, \tilde{\mathbf{z}}_{n}\right)\right)^{T} . \hat{\boldsymbol{\theta}}$ is an unbiased estimator for $\boldsymbol{\theta}$ when there are no measurement errors. However, in case measurement errors exist, which often happen in practice, the OLS estimator is biased.

Wolter and Fuller [23] gave a summary of results concerning the measurement error models. To facilitate our discussion, let:

$$
\begin{aligned}
& \varphi=(\tilde{y}, \tilde{g}(\tilde{\mathbf{x}}, \tilde{\mathbf{z}}))=(y, g(\mathbf{x}, \mathbf{z}))+\boldsymbol{\eta} \\
& \tilde{g}(\tilde{\mathbf{x}}, \tilde{\mathbf{z}})=g(\mathbf{x}, \mathbf{z})+\mathbf{f}=\left(1, \tilde{x}_{1}, \tilde{x}_{2}, \cdots, \tilde{x}_{p}, \tilde{z}_{1}, \tilde{z}_{2}, \cdots\right. \\
& \tilde{z}_{q}, \tilde{x}_{1} \tilde{x}_{1}-\sigma_{w_{1}}^{2}, \tilde{x}_{1} \tilde{x}_{2}, \cdots, \tilde{x}_{p} \tilde{x}_{p}-\sigma_{w_{p}}^{2}, \tilde{x}_{1} \tilde{z}_{1} \\
& \left.\tilde{x}_{1} \tilde{z}_{2}, \cdots, \tilde{x}_{p} \tilde{z}_{q}\right)^{T} \\
& \mathbf{f}=\left(0, w_{x_{1}}, \cdots, w_{z_{q}}, 2 x_{1} w_{x_{1}}+w_{x_{1}}^{2}-\sigma_{w_{x_{1}}}^{2}, x_{1} w_{x_{2}}\right. \\
& \left.+x_{2} w_{x_{1}}+w_{x_{1}} w_{x_{2}}, \cdots, x_{p} w_{z_{q}}+z_{q} w_{x_{p}}+w_{x_{p}} w_{z_{q}}\right) \\
& \boldsymbol{\eta}=(v, \mathbf{f})
\end{aligned}
$$

Note that we have defined $\tilde{g}(\tilde{\mathbf{x}}, \tilde{\mathbf{z}})$ and $\boldsymbol{\eta}$ so that $E(\boldsymbol{\eta})=$ 0. We also define the moment matrix:

$$
M=n^{-1} \sum_{t=1}^{n} \boldsymbol{\varphi}_{t^{\prime}} \boldsymbol{\varphi}_{t}=\left[\begin{array}{ll}
M_{y y} & M_{y x} \\
M_{x y} & M_{x x}
\end{array}\right],
$$

and the covariance matrices:

$$
\Omega_{t}=E\left(\boldsymbol{\eta}_{t}^{T} \boldsymbol{\eta}_{t}\right)=\left[\begin{array}{cc}
\sigma_{v}^{2} & \Omega_{v \mathbf{f}_{t}} \\
\Omega_{\mathbf{f} v_{t}} & \Omega_{\mathbf{f f}_{t}}
\end{array}\right]
$$




$$
\Omega=n^{-1} \sum_{t=1}^{n} \Omega_{t} .
$$

Because the measurement errors are distributed as multivariate normal, the covariance matrix of $\boldsymbol{\eta}_{t}$ is symmetric. Diagonal elements in Eq. (17) are the variance of the corresponding terms, e.g.:

$$
\begin{aligned}
& \operatorname{Var}(\tilde{y})=\sigma_{v}^{2}, \quad \operatorname{Var}\left(\tilde{x}_{i}\right)=\sigma_{w_{x_{i}}}^{2}, \quad \operatorname{Var}\left(\tilde{z}_{i}\right)=\sigma_{w_{z_{i}}}^{2}, \\
& \operatorname{Var}\left(\tilde{x}_{i}^{2}\right)=2 \sigma_{w_{x_{i}}}^{4}+4 x_{i}^{2} \sigma_{w_{x_{i}}}^{2}, \\
& \operatorname{Var}\left(\tilde{x}_{i} \tilde{x}_{j}\right)=\sigma_{w_{x_{i}}}^{2} \sigma_{w_{x_{j}}}^{2}+x_{i}^{2} \sigma_{w_{x_{j}}}^{2}+x_{j}^{2} \sigma_{w_{x_{i}}}^{2} .
\end{aligned}
$$

Other elements are the covariance of different terms, e.g.:

$$
\begin{aligned}
& \operatorname{cov}\left(\tilde{x}_{i}, \tilde{x}_{j}\right)=0, \quad \operatorname{cov}\left(\tilde{x}_{i}, \tilde{x}_{i}^{2}\right)=2 x_{i} \sigma_{w_{x_{i}}}^{2}, \\
& \operatorname{cov}\left(\tilde{x}_{i}, \tilde{x}_{i} \tilde{x}_{j}\right)=x_{j} \sigma_{w_{x_{i}}}^{2}, \quad \operatorname{cov}\left(\tilde{x}_{i}^{2}, \tilde{x}_{i} \tilde{x}_{j}\right)=2 x_{i} x_{j} \sigma_{w_{x_{i}}}^{2}, \\
& \operatorname{cov}\left(\tilde{x}_{i} \tilde{x}_{j}, \tilde{x}_{i} \tilde{x}_{k}\right)=x_{j} x_{k} \sigma_{w_{x_{i}}}^{2}, \quad \operatorname{cov}\left(\tilde{x}_{i} \tilde{x}_{j}, \tilde{x}_{m} \tilde{x}_{k}\right)=0 .
\end{aligned}
$$

The unbiased estimator of $\Omega_{t}$, which is $\hat{\Omega}_{t}$, can be obtained by replacing $x_{i}$ with $\tilde{x}_{i}, x_{i} x_{j}$ with $\tilde{x}_{i} \tilde{x}_{j}$, and $x_{i}^{2}$ with $\tilde{x}_{i}^{2}-\sigma_{x_{i}}^{2}$. Then, the unbiased estimator of $\Omega$ can be constructed by:

$$
\hat{\Omega}=n^{-1} \sum_{t=1}^{n} \hat{\Omega}_{t} .
$$

The unbiased estimator of $\boldsymbol{\theta}$, which is the extended version of Wolter and Fuller [23] for multiple independent variables, is as follows:

$$
\hat{\boldsymbol{\theta}}=\left(\mathbf{M}_{\mathbf{x x}}-\hat{\alpha} \hat{\Omega}_{\mathbf{f f}}\right)^{-1} \mathbf{M}_{\mathbf{x y}},
$$

where $\hat{\alpha}$ is the smallest root of the determinant equation $|\mathbf{M}-\alpha \hat{\Omega}|=0$.

\section{Response surface optimization with measurement error model}

When the levels of the control variables are set with errors, their values become random variables. If $\mathrm{x}$ is the vector of the intended value for the control variables, the factual value $\tilde{\mathbf{x}}$ will be observed as:

$$
\tilde{\mathbf{x}}=\mathbf{x}+\mathbf{w}_{\mathbf{x}} \text {. }
$$

In Eq. (21), $\mathbf{w}_{\mathbf{x}}$ is the vector of measurement errors of the control variables. Naturally, we suppose that the measurement system is unbiased, the covariance of measurement system is $\Sigma_{\mathbf{w}_{\mathbf{x}}}$, and the measurement errors are independent of each other. i.e.:

$$
\begin{aligned}
& \mathbf{w}_{\mathbf{x}} \sim N\left(\mathbf{0}, \boldsymbol{\Sigma}_{\mathbf{w}_{\mathbf{x}}}\right), \\
& \boldsymbol{\Sigma}_{\mathbf{w}_{\mathbf{x}}}=\operatorname{diag}\left(\sigma_{w_{x_{1}}}^{2}, \sigma_{w_{x_{2}}}^{2}, \cdots, \sigma_{w_{x_{p}}}^{2}\right) .
\end{aligned}
$$

Substituting $\tilde{\mathbf{x}}$ for $\mathbf{x}$ of Eq. (21) in Eq. (2) gives:

$$
\begin{aligned}
y=\beta_{\mathbf{0}} & +\boldsymbol{\beta}_{\mathbf{1}}^{\mathbf{T}}\left(\mathbf{x}+\mathbf{w}_{\mathbf{x}}\right)+\boldsymbol{\beta}_{2}^{\mathbf{T}} \mathbf{z}+\left(\mathbf{x}+\mathbf{w}_{\mathbf{x}}\right)^{\mathbf{T}} \mathbf{B}_{1}\left(\mathbf{x}+\mathbf{w}_{\mathbf{x}}\right) \\
& +\left(\mathbf{x}+\mathbf{w}_{\mathbf{x}}\right)^{\mathbf{T}} \mathbf{B}_{2} \mathbf{z}+\varepsilon .
\end{aligned}
$$

Hence, the expectation and variance of the responses can be derived

$$
\begin{aligned}
E_{\mathbf{w}_{\mathbf{x}}, \mathbf{z}}(y)= & \beta_{0}+\boldsymbol{\beta}_{1}^{T} \mathbf{x}+\operatorname{tr}\left(\mathbf{B}_{1} \Sigma_{\mathbf{w}_{\mathbf{x}}}\right)+\mathbf{x}^{T} \mathbf{B}_{1} \mathbf{x}, \quad(23) \\
\operatorname{Var}_{\mathbf{w}_{\mathbf{x}}, \mathbf{z}}(y)= & E_{x}\left[\operatorname{var}_{z}(y)\right]+\operatorname{var}_{x}\left[E_{z}(y)\right] \\
& =E_{x}\left[\left(\boldsymbol{\beta}_{2}^{T}+\mathbf{x}^{T} \mathbf{B}_{2}\right) \boldsymbol{\Sigma}_{z}\left(\boldsymbol{\beta}_{2}^{T}+\mathbf{x}^{T} \mathbf{B}_{2}\right)^{T}\right] \\
& +\operatorname{var}_{x}\left[\beta_{0}+\boldsymbol{\beta}_{1}^{T} x+\mathbf{x}^{T} \mathbf{B}_{1} \mathbf{x}\right]+\sigma^{2} \\
& =\operatorname{tr}\left(\mathbf{B}_{2}^{T} \Sigma_{\mathbf{w}_{\mathbf{x}}} \mathbf{B}_{2} \boldsymbol{\Sigma}_{z}^{T}\right)+\left(\boldsymbol{\beta}_{2}^{T}+\mathbf{x}^{T} \mathbf{B}_{2}\right) \\
& \left(\boldsymbol{\Sigma}_{\mathbf{z}}^{T} \mathbf{B}_{2}^{T} \mathbf{x}+\boldsymbol{\Sigma}_{\mathbf{z}} \beta_{2}\right)+2 \operatorname{tr}\left(\mathbf{B}_{1} \Sigma_{\mathbf{w}_{\mathbf{x}}} \mathbf{B}_{1} \Sigma_{\mathbf{w}_{\mathbf{x}}}\right) \\
& +4\left(\mathbf{x}+\frac{1}{2} \mathbf{B}_{1}^{-1} \boldsymbol{\beta}_{1}\right)^{T} \mathbf{B}_{1} \Sigma_{\mathbf{w}_{\mathbf{x}}} \mathbf{B}_{1} \\
& \left(\mathbf{x}+\frac{1}{2} \mathbf{B}_{1}^{-1} \boldsymbol{\beta}_{1}\right)+\sigma^{2} .
\end{aligned}
$$

In Eqs. (23) and (24), $E_{\mathbf{w}_{\mathbf{x}}, \mathbf{z}}(y)$ represents the expectation of responses $y$ taken over the distribution of random variables $\mathbf{w}_{\mathbf{x}}$ and $\mathbf{z}$, and $\operatorname{Var}_{\mathbf{w}_{\mathbf{x}}, \mathbf{z}}(y)$ represents the variance of response $y$ taken over the distribution of random variables $\mathbf{w}_{\mathbf{x}}$ and $\mathbf{z}$.

Then, the conditional objective function can be expressed as follows:

$$
\begin{aligned}
& J_{\mathbf{w}_{\mathbf{x}}, \mathbf{z}}(\mathbf{x})=E(L)= \\
& \left\{\begin{array}{l}
\left(E_{\mathbf{w}_{\mathbf{x}}, \mathbf{z}}(y)-\tau\right)^{2}+\operatorname{Var}_{\mathbf{w}_{\mathbf{x}}, \mathbf{z}}(y) \\
E_{\mathbf{w}_{\mathbf{x}}, \mathbf{z}}(y)^{2}+\operatorname{Var}_{\mathbf{w}_{\mathbf{x}}, \mathbf{z}}(y) \\
E_{\mathbf{w}_{\mathbf{x}}, \mathbf{z}}\left(y^{\prime}\right)^{2}+\operatorname{Var}_{\mathbf{w}_{\mathbf{x}}, \mathbf{z}}\left(y^{\prime}\right)
\end{array}\right.
\end{aligned}
$$

The optimal setting of the control variables will be obtained to minimize the expected quality loss as follows:

$$
\mathbf{x}^{*}=\min _{\mathbf{x} \in \Theta} J_{\mathbf{w}_{\mathbf{x}}, \mathbf{z}}(\mathbf{x}) .
$$

If there are no measurement errors, i.e., $\boldsymbol{\Sigma}_{\mathbf{w}_{\mathbf{x}}}=\mathbf{0}$, the optimal setting of control variables will be the same as those obtained by the traditional robust parameter design.

\section{Example}

In this section, the proposed method is illustrated in detail using a turning operation process case study introduced in Kirby et al. [33]. A typical turning operation produces parts that have critical features requiring specific surface roughness. The objective 
Table 1. Factors, codes, and level values used for the orthogonal array.

\begin{tabular}{lcccc}
\hline Parameter & Code & $\mathbf{- 1}$ & $\mathbf{0}$ & $\mathbf{1}$ \\
\hline Control variables & & & & \\
Spindle speed (rpm) & $x_{1}$ & 1500 & 2250 & 3000 \\
Feed rate (ipr) & $x_{2}$ & 0.004 & 0.008 & 0.012 \\
Depth of cut (in) & $x_{3}$ & 0.010 & 0.020 & 0.030 \\
Tool radius (in) & $x_{4}$ & 0.008 & 0.016 & 0.032 \\
Noise variables & & & & \\
Tool number & $z_{1}$ & 1 & & 2 \\
Temperature range $\left({ }^{\circ} \mathrm{F}\right)$ & $z_{2}$ & $65-75$ & & $95-100$ \\
Response variable & & & & \\
Surface roughness $(\mu \mathrm{in})$ & $Y$ & & & \\
\hline
\end{tabular}

of the experiment is to reduce surface roughness by controlling four control variables. The control variables are spindle speed $\left(x_{1}\right)$, feed rate $\left(x_{2}\right)$, depth of cut $\left(x_{3}\right)$, and tool nose radius $\left(x_{4}\right)$. At the same time, tool inserts $\left(z_{1}\right)$ and room temperature $\left(z_{2}\right)$ are noise variables that cannot be controlled. A modified orthogonal array is created using the orthogonal array $L_{9}\left(3^{4}\right)$ and the selected parameters, as shown in Table 1. A total of 36 experiments are run and the design and experimental data are given in Table 2. The control variables and noise variables are coded from -1 to 1 . By supposing that the variance of noise variables is equal to $1 / 3$ $\left(\frac{U S L-L S L}{6}\right)$, most of noise variables range from -1 to 1 .

To fit the empirical model, terms up to the second order are taken into account and significant terms are included in the model for further analysis. Based on the experimental data, a stepwise regression is used to fit the turning process model by using $\alpha=0.05$ for both enter and remove tests in MINITAB. It is assumed that there are some measurement errors in the design stage and all variables are independently and identically distributed with the variance of measurement errors ranging from 0 to 0.07 . We can get the estimate of the parameters by the method proposed in Section 3. The results are given in Table 3 .

From the above results, the control variable $x_{3}$ (depth of cut) is not a significant factor in reducing the surface roughness such that it can be set according to economic advantages during the production. At the same time, the noise variable $z_{2}$ (room temperature) is also not significant in reducing the surface roughness. In addition, the response surface model can be obtained as follows:

$$
y=\beta_{\mathbf{0}}+\boldsymbol{\beta}_{\mathbf{1}}^{\mathbf{T}} \mathbf{x}+\boldsymbol{\beta}_{2}^{\mathbf{T}} \mathbf{z}+\mathbf{x}^{\mathbf{T}} \mathbf{B}_{1} \mathbf{x}+\mathbf{x}^{\mathbf{T}} \mathbf{B}_{2} \mathbf{z},
$$

where $\mathbf{x}=\left[x_{1}, x_{2}, x_{4}\right]^{T}$ and $\mathbf{z}=z_{1}$. When there are no measurement errors, that is, the variance of measurement errors of all variables equals 0 , the estimated parameters are:
Table 2. Data of the designed experiment.

\begin{tabular}{|c|c|c|c|c|c|c|c|}
\hline Order & $x_{1}$ & $x_{2}$ & $x_{3}$ & $\boldsymbol{x}_{4}$ & $z_{1}$ & $z_{2}$ & $\boldsymbol{Y}$ \\
\hline 1 & -1 & -1 & -1 & -1 & -1 & -1 & 47 \\
\hline 2 & -1 & 0 & 0 & 0 & -1 & -1 & 137 \\
\hline 3 & -1 & 1 & 1 & 1 & -1 & -1 & 145 \\
\hline 4 & 0 & -1 & 0 & 1 & -1 & -1 & 32 \\
\hline 5 & 0 & 0 & 1 & -1 & -1 & -1 & 159 \\
\hline 6 & 0 & 1 & -1 & 0 & -1 & -1 & 230 \\
\hline 7 & 1 & -1 & 1 & 0 & -1 & -1 & 44 \\
\hline 8 & 1 & 0 & -1 & 1 & -1 & -1 & 64 \\
\hline 9 & 1 & 1 & 0 & -1 & -1 & -1 & 244 \\
\hline 10 & -1 & -1 & -1 & -1 & -1 & 1 & 47 \\
\hline 11 & -1 & 0 & 0 & 0 & -1 & 1 & 137 \\
\hline 12 & -1 & 1 & 1 & 1 & -1 & 1 & 147 \\
\hline 13 & 0 & -1 & 0 & 1 & -1 & 1 & 31 \\
\hline 14 & 0 & 0 & 1 & -1 & -1 & 1 & 162 \\
\hline 15 & 0 & 1 & -1 & 0 & -1 & 1 & 214 \\
\hline 16 & 1 & -1 & 1 & 0 & -1 & 1 & 49 \\
\hline 17 & 1 & 0 & -1 & 1 & -1 & 1 & 69 \\
\hline 18 & 1 & 1 & 0 & -1 & -1 & 1 & 240 \\
\hline 19 & -1 & -1 & -1 & -1 & 1 & -1 & 61 \\
\hline 20 & -1 & 0 & 0 & 0 & 1 & -1 & 124 \\
\hline 21 & -1 & 1 & 1 & 1 & 1 & -1 & 117 \\
\hline 22 & 0 & -1 & 0 & 1 & 1 & -1 & 26 \\
\hline 23 & 0 & 0 & 1 & -1 & 1 & -1 & 178 \\
\hline 24 & 0 & 1 & -1 & 0 & 1 & -1 & 207 \\
\hline 25 & 1 & -1 & 1 & 0 & 1 & -1 & 50 \\
\hline 26 & 1 & 0 & -1 & 1 & 1 & -1 & 52 \\
\hline 27 & 1 & 1 & 0 & -1 & 1 & -1 & 248 \\
\hline 28 & -1 & -1 & -1 & -1 & 1 & 1 & 64 \\
\hline 29 & -1 & 0 & 0 & 0 & 1 & 1 & 130 \\
\hline 30 & -1 & 1 & 1 & 1 & 1 & 1 & 116 \\
\hline 31 & 0 & -1 & 0 & 1 & 1 & 1 & 36 \\
\hline 32 & 0 & 0 & 1 & -1 & 1 & 1 & 171 \\
\hline 33 & 0 & 1 & -1 & 0 & 1 & 1 & 210 \\
\hline 34 & 1 & -1 & 1 & 0 & 1 & 1 & 40 \\
\hline 35 & 1 & 0 & -1 & 1 & 1 & 1 & 46 \\
\hline 36 & 1 & 1 & 0 & -1 & 1 & 1 & 247 \\
\hline
\end{tabular}

$$
\begin{aligned}
\beta_{0}= & 139.72, \quad \boldsymbol{\beta}_{1}=[-5.71,69.89,-48]^{T}, \quad \boldsymbol{\beta}_{2}=-4.17 \\
\mathbf{B}_{1}= & {[-16.2,0,-2.45 ; 0,0,-10.755 ;-2.45,-10.755} \\
& \quad-16.46] \\
\mathbf{B}_{2}= & {[0,-8.5,-13.75]^{T} . }
\end{aligned}
$$

From Table 4, it can be seen that even though the errors 
Table 3. Parameter estimation under different magnitudes of measurement errors.

\begin{tabular}{ccccccccc}
\hline \multirow{2}{*}{ Term } & \multicolumn{7}{c}{ Variance of measurement errors } \\
\cline { 2 - 9 } & $\mathbf{0}$ & $\mathbf{0 . 0 1}$ & $\mathbf{0 . 0 2}$ & $\mathbf{0 . 0 3}$ & $\mathbf{0 . 0 4}$ & $\mathbf{0 . 0 5}$ & $\mathbf{0 . 0 6}$ & $\mathbf{0 . 0 7}$ \\
\hline Constant & 139.72 & 141.88 & 144.71 & 148.68 & 155.08 & 136.37 & 173.81 & 214.76 \\
$x_{1}$ & -5.71 & -6.46 & -7.31 & -8.24 & -8.86 & -29.76 & -16.44 & -19.30 \\
$x_{2}$ & 69.89 & 71.60 & 73.51 & 75.65 & 77.85 & 91.74 & 87.04 & 92.23 \\
$x_{4}$ & -48.00 & -49.00 & -50.08 & -51.25 & -52.52 & -53.93 & -55.51 & -57.31 \\
$z_{1}$ & -4.17 & -4.34 & -4.52 & -4.72 & -4.93 & -5.17 & -5.43 & -5.73 \\
$x_{1} x_{1}$ & -16.20 & -17.63 & -19.42 & -21.87 & -26.14 & 14.36 & -25.76 & -32.63 \\
$x_{4} x_{4}$ & -16.46 & -18.65 & -21.57 & -25.63 & -31.69 & -41.69 & -61.30 & -117.18 \\
$x_{1} x_{4}$ & -4.90 & -3.19 & -1.16 & 1.25 & 3.76 & 27.90 & 17.46 & 25.25 \\
$x_{2} x_{4}$ & -21.51 & -22.81 & -24.27 & -25.84 & -26.76 & -65.26 & -40.08 & -44.75 \\
$x_{2} z_{1}$ & -8.50 & -8.99 & -9.54 & -10.19 & -10.94 & -11.84 & -12.91 & -14.24 \\
$x_{4} z_{1}$ & -13.75 & -14.54 & -15.44 & -16.48 & -17.70 & -19.15 & -20.89 & -23.03 \\
\hline
\end{tabular}

Table 4. Optimal setting and Expected Quality Loss (EQL) with different magnitudes of measurement errors in the production stage.

\begin{tabular}{cccccc}
\hline & \multicolumn{3}{c}{ True optimal setting } & $\begin{array}{c}\text { Quality loss } \\
\text { under true } \\
\text { optimal setting }\end{array}$ & $\begin{array}{c}\text { Quality loss } \\
\text { under initial } \\
\text { optimal setting }\end{array}$ \\
\cline { 2 - 4 } Variance & $\boldsymbol{A}$ & $\boldsymbol{B}$ & $\boldsymbol{D}$ & & 29.21 \\
\hline 0 & 1.000 & -1.000 & 0.991 & 29.21 & 112.95 \\
0.01 & 1.000 & -1.000 & 0.982 & 112.68 & 197.22 \\
0.02 & 1.000 & -1.000 & 0.974 & 196.13 & 282.01 \\
0.03 & 1.000 & -1.000 & 0.966 & 279.57 & 367.32 \\
0.04 & 0.932 & -1.000 & 1.000 & 362.16 & 453.16 \\
0.05 & 0.914 & -1.000 & 1.000 & 443.97 & 539.52 \\
0.06 & 0.896 & -1.000 & 1.000 & 525.23 & 626.41 \\
0.07 & 0.877 & -1.000 & 1.000 & 605.94 & \\
\hline
\end{tabular}

in independent variables occur at a low level, they have a great impact on model fitting. As the variance of the measurement errors increases, the estimated coefficients of the parameters deviate further from those obtained under no measurement errors case.

Since the experimenters are interested in minimizing surface roughness, the optimization function is the STB type expected loss. In the surface operation process, variance of measurement errors can be obtained using measurement system analysis.

If there are no measurement errors in the design stage, meaning the variables are measured with precision in applying design of experiments, errors can only occur in the production stage. As can be seen in Eq. (21), optimal setting $x^{*}$ cannot be set precisely in production. The actual input value is $\tilde{\mathbf{x}}^{*}$ due to measurement errors. Assume that the measurement system is unbiased and the variance ranges from 0 to 0.07 , i.e., $\sigma_{\mathbf{w}_{\mathbf{x}}}^{2} \in[0,0.07]$. When the variance of measurement system equals 0 , the optimal setting can be obtained by the traditional responses surface optimization approach proposed by Welch et al. [27]. The optimization problem in Eq. (26) will be given in Eq. (28), which would be solved by genetic algorithm using MATLAB:

$$
\begin{aligned}
& \operatorname{Min}_{\mathbf{x}} J_{\mathbf{w}, \mathbf{z}}(\mathbf{x})=u_{\mathbf{w}, \mathbf{z}}^{2}(\mathbf{x})+\sigma_{\mathbf{w}, \mathbf{z}}^{2}(\mathbf{x}) \\
& \text { s.t. } \mathbf{x} \in[-\mathbf{1}, \mathbf{1}]
\end{aligned}
$$

Optimization results are presented in Table 4. As can be seen in Table 4 , the initial optimal setting $\left(x_{1}, x_{2}, x_{4}\right)=(1.000,-1.000,0.970)$ is calculated by the traditional response surface approach when there are no measurement errors. The actual optimal setting changes along with the variance of measurement errors of control variables. Table 4 also gives the actual optimal setting and the corresponding expected quality loss with respect to different measurement errors. Figure 1 shows the comparison of the performances 


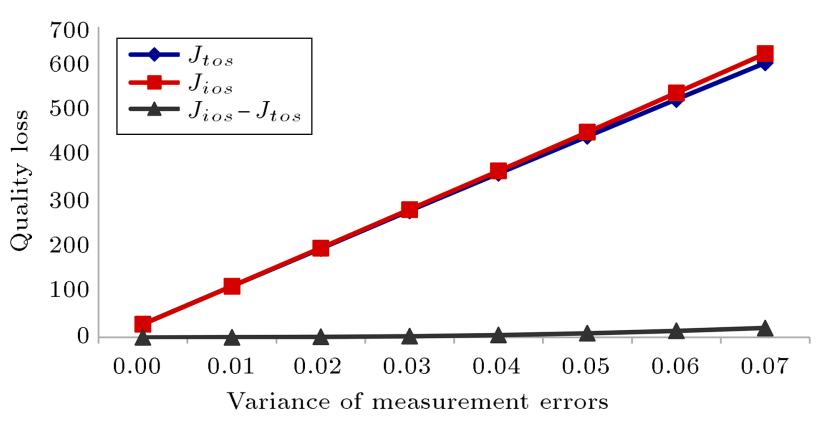

Figure 1. Comparison of expected quadratic losses with and without consideration of measurement errors in the production stage.

of the estimators with and without consideration of measurement errors in the production stage. $J_{\text {ios }}$ and $J_{\text {tos }}$ are the expected quality losses under initial optimal setting obtained with no measurement error assumption, and under true optimal setting obtained considering measurement errors in the production stage, respectively. If the initial optimal setting with no measurement errors assumption is still used as the process input with measurement errors, the expected quality loss will be more significant than that obtained with measurement errors consideration. However, the gaps between the two expected quality losses are small and can be ignored.

Suppose that the levels of the control variables can be measured with errors in the design stage. From Table 3 we find that measurement errors have a great impact on model fitting when they occur in the design stage. If measurement errors occur in both the design and production stages, the true optimum condition may be different from the initial optimal setting. We suppose that the distributions of measurement errors in the design stage are the same as the ones in the production stage when the variance of measurement errors ranges from 0 to 0.07 . That is, the diagonal elements of covariance matrix of $M V N\left\{0,\left[\begin{array}{cc}\sigma_{v}^{2} & 0 \\ 0 & \Sigma_{\mathbf{w}}\end{array}\right]\right\}$ are the same. Table 5 presents the actual optimal setting and the corresponding expected quality loss under different magnitudes of measurement error. In case of measurement errors existing, if the initial optimal setting obtained without measurement errors assumption is still used, the expected quality loss will be much more considerable than that under the optimal setting with measurement errors. This event happens even when the variance of the measurement errors is very small. If there are measurement errors in the design and the production stage, it can be found that not only are the regression coefficients poorly estimated by the usual OLS, but also the prediction property is severely damaged. At the same time, the optimum operating condition of control variables is located far from the true optimum condition obtained by the

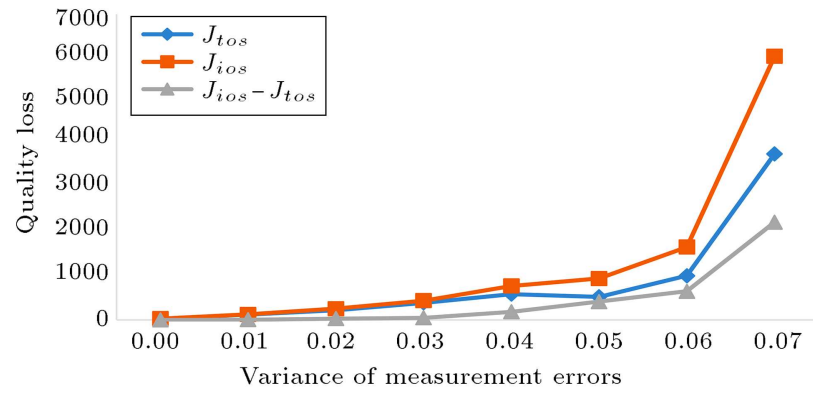

Figure 2. Comparison of expected quadratic losses with and without consideration of measurement errors in the design stage.

unbiased estimation method of Eq. (20). Figure 2 shows the comparison of the performances of the estimators with and without consideration of measurement errors in the design stage. $J_{\text {ios }}$ is quality loss under initial optimal setting, and $J_{\text {tos }}$ is quality loss under true optimal setting obtained considering measurement errors. When the variance of measurement errors is larger than 0.04, considering measurement errors is imperative. Upon comparing Figures 1 and 2, we can see that measurement errors in the production stage have a very negligible impact on the expected loss, while the errors would be highly influential in the design stage. As seen in Figure 2 and Table 5, the expected quality loss soars up when the magnitude of measurement errors is 0.07 or larger.

\section{Discussion and concluding remarks}

To find the optimal setting of the control variables to ensure a robust design based on response surface methodology, design engineers usually do not consider measurement errors. However, in reality, measurement errors exist, yield poor estimation of the regression coefficients, and damage the prediction capability. This paper proposed an approach to properly estimate a response surface model, which takes into account measurement errors both in the design and production stages. We presented a modeling method when there were some measurement errors in the design stage. Location and dispersion performances of quality characteristics were given and then, optimal setting of control variables was derived in the production stage. With a turning operation case study, the performances of these settings were compared with the performance of the setting obtained with no measurement errors assumption. Analysis results show that errors in variables are needed to be taken into account for parameter estimation and prediction to reduce the expected quality loss in the design stage.

\section{Acknowledgement}

This work was supported by the National Natural 
Table 5. Optimal setting and Expected Quality Loss (EQL) with different magnitudes of measurement errors in the design stage.

\begin{tabular}{|c|c|c|c|c|c|}
\hline \multirow[b]{2}{*}{ Variance } & \multicolumn{3}{|c|}{$\begin{array}{l}\text { True optimal } \\
\text { setting }\end{array}$} & \multirow[b]{2}{*}{$\begin{array}{l}\text { EQL under true } \\
\text { optimal setting }\end{array}$} & \multirow[b]{2}{*}{$\begin{array}{c}\text { EQL under initial } \\
\text { optimal setting }\end{array}$} \\
\hline & $A$ & $B$ & $D$ & & \\
\hline 0 & 1.000 & -1.000 & 0.991 & 29.23 & 29.23 \\
\hline 0.01 & 1.000 & -1.000 & 0.953 & 117.26 & 123.55 \\
\hline 0.02 & 1.000 & -1.000 & 0.911 & 217.02 & 247.16 \\
\hline 0.03 & -1.000 & -1.000 & 1.000 & 376.22 & 424.30 \\
\hline 0.04 & -1.000 & -1.000 & 0.931 & 569.72 & 750.06 \\
\hline 0.05 & 0.275 & -1.000 & 1.000 & 508.44 & 913.49 \\
\hline 0.06 & 1.000 & -1.000 & 0.756 & 974.17 & 1607.07 \\
\hline 0.07 & 1.000 & -1.000 & -0.522 & 3660.60 & 5816.29 \\
\hline
\end{tabular}

Science Foundation of China (No. 71771121, 71371099, 71471088, and 71931006), which is gratefully acknowledged.

\section{References}

1. Taguchi, G., Introduction to Quality Engineering: Designing Quality into Products and Processes, Asian Productivity Organization: Tokyo (1986).

2. Taguchi, G., System of Experimental Design: Engineering Methods to Optimize Quality and Minimize Costs, UNIPUB/Kraus International Publication (1988).

3. Nair, V.N. “Taguchi's parameter design: a panel discussion", Technometrics, 34(2), pp. 127-161 (1992).

4. Phadke, M.S., Quality Engineering Using Robust Design, Prentice Hall: New Jersey (1989).

5. Wu, J.C.F. and Hamada, M.S., Experiments: Planning, Analysis, and Optimization, 2th Edn., Wiley: New York (2011).

6. Myers, R.H., Montgomery, D.C., and Anderson-Cook, C.M. "Response surface methodology: process and product optimization using designed experiments", 3rd Edn., Wiley: New York (2009).

7. Ouyang, L.H., Ma, Y.Z., Chen, J., et al. "Robust optimisation of Nd: YLF laser beam micro-drilling process using Bayesian probabilistic approach", International Journal of Production Research, 54(21), pp. 6644-6659 (2016).

8. Zhou, X.J., Ma, Y.Z., Tu, Y.L., et al. "Ensemble of surrogates for dual response surface modeling in robust parameter design", Quality \& Reliability Engineering International, 29(2), pp. 173-197 (2013).

9. Emdadi, L., Nasernajad, B., Shokrgozar, S.T., et al. "Optimization of withering time and fermentation conditions during the manufacture of black tea using a response surface methodology", Scientia Iranica, 16(1), pp. 61-68 (2009).
10. Bellucci, J.P. and Jr, K.W.B. "The use of nested desirability functions and quality indices for multiresponse robust parameter design problems", Quality Engineering, 29(4), pp. 573-587 (2017).

11. Zhai, Q., Ye, Z.S., Yang, J., et al. "Measurement errors in degradation-based burn-in", Reliability Engineering \& System Safety, 150, pp. 126-135 (2016).

12. Grau, D. "Lower confidence bound for capability indices with one-sided tolerance processes and measurement errors", International Journal of Quality Engineering \& Technology, 2(3), pp. 212-228 (2013).

13. Rakhmawati, D.Y, Wu, C.W, Yang, C.L. "Performance evaluation of processes with asymmetric tolerances in the presence of gauge measurement errors", Communications in Statistics, 45(10), pp. 3011-3026 (2016).

14. Loken, E and Gelman, A. "Measurement error and the replication crisis", Science, 355(6325), pp. 584585 (2017).

15. Feng, C.X.J., Saal, A.L., Salsbury, J.G., et al. "Design and analysis of experiments in CMM measurement uncertainty study", Precision Engineering, 31(2), pp. 94-101 (2007).

16. Giovagnoli, A. and Romano D. "Robust design via simulation experiments: a modified dual response surface approach", Quality and Reliability Engineering International, 24(4), pp. 401-416 (2008).

17. Box, G.E.P. "The effects of errors in the factor levels and experimental design", Technometrics, 5(2), pp. 247-262 (1963).

18. Draper, N.R. and Beggs W.J. "Errors in the factor levels and experimental design", The Annals of Mathematical Statistics, 42, pp. 46-58 (1971).

19. Steiner, S.H. and Hamada, M. "Making mixtures robust to noise and mixing measurement errors", Journal of Quality Technology, 29(4), pp. 441-450 (1997).

20. Donev, A.N. "Design of experiments in the presence of errors in factor levels", Journal of Statistical Planning and Inference, 126(2), pp. 569-585 (2004). 
21. Zhong, J., Shi, J., and Wu, C.F.J. "Design of DOEbased automatic process controller with consideration of model and observation uncertainties", IEEE Transactions on Automation Science and Engineering, 7(2), pp. 266-273 (2010).

22. Ardakani, M.K. "The impacts of errors in factor levels on robust parameter design optimization", Quality \& Reliability Engineering International, 32(5), pp. 19291944 (2016).

23. Wolter, K.M. and Fuller, W.A. "Estimation of the quadratic errors-in-variables model", Biometrika, 69(1), pp. 175-182 (1982).

24. Chang, K.C., Han, C.P., and Hawkins, D.L. "Improved estimators of regression coefficients in measurement error models", Journal of Statistical Computation \& Simulation, 39(1-2), pp. 21-35 (2016).

25. Zhang, X., Wang, H., Ma, Y., et al. "Linear model selection when covariates contain errors", Journal of the American Statistical Association, 112(520), pp. 1553-1561 (2017).

26. Firpo. S., Galvao, A.F., and Song S. "Measurement errors in quantile regression models", Journal of Econometrics, 198(1), pp. 146-164 (2017).

27. Welch, W., Yu, T., Kang, S.M., et al. "Computer experiments for quality control by parameter design", Journal of Quality Technology, 22(1), pp. 15-22 (1990).

28. Vining, G.G. and Myers, R.H. "Combining Taguchi and response surface philosophies: A dual response approach", Journal of Quality Technology, 22(1), pp. 38-45 (1990)

29. Myers, R.H., Khuri, A.I., and Vining, G. "Response surface alternatives to the Taguchi robust parameter design approach", The American Statistician, 46(2), pp. 131-139 (1992).

30. Myers, W.R., Brenneman, W.A., and Myers, R.H. "A dual-response approach to robust parameter design for a generalized linear model", Journal of Quality Technology, 37(2), pp. 130-138 (2005).

31. Ames, A.E., Mattucci, N., and MacDonald, S., et al. "Quality loss functions for optimization across multiple response surfaces", Journal of Quality Technology, 29(3), pp. 339-346 (1997).

32. Murphy, T.E., Tsui, K.L., and Allen, J.K. "A review of robust design methods for multiple responses", Research in Engineering Design, 15(4), pp. 201-215 (2005).
33. Kirby, E.D., Zhang, Z., Chen, J.C., et al. "Optimizing surface finish in a turning operation using the Taguchi parameter design method", The International Journal of Advanced Manufacturing Technology, 30(11-12), pp. 1021-1029 (2006).

\section{Biographies}

Feng $\mathbf{W u}$ is currently a lecturer at the Department of Industrial Engineering at Anhui Polytechnic University. He received the BS in Business Management from Nanjing University of Aeronautics and Astronautics of China. He earned his PhD in Management Science and Engineering from Nanjing University of Science and Technology, China. His research interests include statistical process control, process modeling, and design of experiments.

Jian-Jun Wang is an Associate Professor at the Department of Management Science and Engineering at Nanjing University of Science and Technology. He received his BSc in Applied Mathematics from Jishou University, China and his MSc in Applied Statistics from Hunan University. He earned his PhD in Quality Engineering from Nanjing University of Science and Technology, China. He is a member of QSR and INFORMS and a senior member of Chinese Society of Optimization, Overall Planning and Economical Mathematics. He has authored over 50 refereed journal publications and is a reviewer of several international journals such as EJOR, IJPR, CIE, and QTQM. His research interests include parameter design and optimization, Bayesian statistics and modeling, and industrial statistical and data analysis.

Yi-Zhong $\mathbf{M a}$ is a Professor at the Department of Management Science and Engineering at Nanjing University of Science and Technology. He received his BSc in Applied Mathematics from Huazhong Normal University, Wuhan, China, MSc in Quality Engineering, and $\mathrm{PhD}$ in Control Science from Northwestern Polytechnical University, China. $\mathrm{He}$ is the executive director of several associations such as Quality Society of China (QSC), Society of Management Science and Engineering of China (SMSEC), and Chinese Society of Optimization, Overall Planning and Economical Mathematics. His research interests include quality engineering and quality management. 